\title{
La importancia de la modificación de la conducta en la salud pública
}

Muchos de los grandes problemas en salud de hoy en día pudieran ser prevenibles, incluyendo la obesidad, la diabetes, las enfermedades asociadas al consumo del tabaco y el alcohol, como son la enfermedades cardiovasculares o el cáncer. Sin embargo, la modificación de la conducta en "pro" de nuestra salud, no es un problema menor. En 2015, las Naciones Unidas se promulgaron a favor de brindar su apoyo a la salud mental para entrar como parte de las metas del desarrollo sustentable dentro de las enfermedades no transmisibles.

Durante años, las organizaciones de salud no han reconocido por completo el impacto de los daños causados por la enfermedad mental. Las enfermedades mentales, contribuyen con una pérdida de $37 \%$ de los años de vida saludables, de todas las enfermedades no transmisibles, según las Naciones Unidas (1). Esto se refleja en un acortamiento de la expectativa de vida, así como una disminución de los años de vida productivos de una persona.

El mundo debe promover la salud mental y el bienestar del ser humano, sin embargo, hacer la labor de promoción solamente, no es suficiente. Una gran parte de las sociedades, incluso las mas desarrolladas, destinan una porción muy limitada de sus servicios a la salud mental y únicamente cuando hay eventos particulares detonantes, como tiroteos en escuelas causados por alguien con enfermedad mental, se dedica mas atención mediática, sin embargo, esto raramente lleva a un incremento real del financiamiento sostenido para la salud mental dentro de los servicios generales de salud. Por otro lado, persiste el debate sobre si muchos de estos problemas, son dilemas de la volición o bien conflictos morales, y no el resultado de alteraciones complejas en el funcionamiento del sistema nervioso central, a pesar de existir una abrumadora cantidad de datos que respaldan estos hechos (2). Se ha estimado que, en países desarrollados, hay 9 profesionales de la salud mental por cada100,000 habitantes; en los países en desarrollo y en países pobres, solo hay uno por 100,000. Esta enorme disparidad, aunado a la cifra en sí misma, refleja la insuficiente cobertura del problema.

A pesar de existir una gran cantidad de evidencia científica, las personas encuentran difícil hacer la analogía de tratar a la depresión como la enfermedad cardiovascular, en parte, por la falta de marcadores clínicos que señalen, de manera concurrente, al diagnostico por el médico de anormalidades que reflejen la patología. Sin embargo, las evidencias provenientes de los campos de la neuro-imagenología, la genética, la neuro-bioquímica, la electrofisiología, la neuropsicología y la farmacología, al comparar grupos de pacientes contra controles, claramente encuentran diferencias entre casos y no casos; pero, por otra parte, la existencia de marcadores biomédicos que nos ayuden a señalar el estado clínico del paciente a nivel diagnóstico, todavía no se encuentran debidamente validados (3). Por otro lado, la eficacia de los métodos de modificación de la conducta como son los tratamientos farmacológicos $(4,5)$, los psicoterapéuticos (6), e, incluso, la estimulación directa del cerebro (7) han sido plenamente validados científicamente. El problema de transferir este tipo de estrategias a los servicios de salud general es el poder incorporar adecuadamente al equipo multidisciplinario de la salud mental dentro de la inter-disciplina que representa la salud en general para los diferentes abordajes, como el cardiovascular, nutricional, endocrinológico, oncológico, etc. Es un reto complejo, pero que, dado la relevancia a nivel de varios ejes que tiene el bienestar de la salud, 


\section{Nicolini-Sánchez}

mental es una inversión importante hacia la mejora de la salud pública en el futuro de nuestras sociedades.

Humberto Nicolini Sánchez

Subdirector de Investigación Básica, Instituto Nacional de Medicina Genómica

Sistema Nacional de Investigadores, nivel III

Miembro Titular de la Academia Nacional de Medicina

\section{REFERENCIAS}

1. Editorial. Mind Matters. Nature; 2016 Apr; pp6.

2. Sekar A, Bialas AR, de Rivera H, Davis A, Hammond TR, Kamitaki N, et al; Schizophrenia Working Group of the Psychiatric Genomics Consortium, Daly MJ, Carroll MC, Stevens B, McCarroll SA. Schizophrenia risk from complex variation of complement component 4. Nature. 2016 Feb 11;530(7589):177-83

3. Niculescu AB, Levey DF, Phalen PL, LeNiculescu H, Dainton HD, Jain N, et al Understanding and predicting suicidality using a combined genomic and clinical risk assessment approach. Mol Psychiatry. 2015 Nov;20(11):1266-85.

4. Liebowitz MR, Salman E, Nicolini H, Rosenthal N, Hanover R, Monti L. Effect of an Acute Intranasal Aerosol Dose of PH94B on Social and Performance Anxiety in Women With Social Anxiety Disorder. Am J Psychiatry. 2014 Jun;171(6):675-82

5. Van Ameringen M, Simpson W, Patterson, Dell'Osso B, Fineberg N, Hollander E, et al Pharmacological treatment strategies in obsessive compulsive disorder: A crosssectional view in nine international OCD centers. J Psychopharmacol 2014 Jan 15;28(6):596-602

6. Tyrer P, Salkovskis P, Tyrer H, Dupont S, Murphy D. Cognitive behaviour therapy for health anxiety.Lancet. 2014 Ene;383(9925):1295-1296

7. Jiménez F, Velasco F, Salín-Pascual R, Hernández J, Nicolini H. A Patient with resistant major depression disorder treated with deep brain stimulation in the inferior thalamic peduncle. Neurosurgery 2005 Sept; 57(3): 585-583. 